\title{
Lifetime cost-effectiveness analysis of first- line dialysis modalities for patients with end-stage renal disease under peritoneal dialysis first policy
}

Carlos K. H. Wong ${ }^{1 *} \mathbb{D}$, Julie Chen ${ }^{1,2} \mathbb{D}$, Samuel K. S. Fung ${ }^{3}$, Maggie Mok ${ }^{4}$, Yuk lun Cheng ${ }^{5}$, Irene Kong ${ }^{3}$, Wai Kei Lo ${ }^{4}$, Sing Leung Lui ${ }^{4}$, T. M. Chan ${ }^{6}$ (D) and Cindy L. K. Lam $^{1}$ (D)

\begin{abstract}
Background: This study aimed to determine the lifetime cost-effectiveness of first-line dialysis modalities for endstage renal disease (ESRD) patients under the "Peritoneal Dialysis First" policy.

Methods: Lifetime cost-effectiveness analyses from both healthcare provider and societal perspectives were performed using Markov modelling by simulating at age 60. Empirical data on costs and health utility scores collected from our studies were combined with published data on health state transitions and survival data to estimate the lifetime cost, quality-adjusted life-years (QALYS) and cost-effectiveness of three competing dialysis modalities: peritoneal dialysis (PD), hospital-based haemodialysis (HD) and nocturnal home HD.
\end{abstract}

Results: For cost-effectiveness analysis over a lifetime horizon from the perspective of healthcare provider, hospitalbased HD group (lifetime cost USD\$142,389; 6.58 QALYs) was dominated by the PD group (USD\$76,915; 7.13 QALYS). Home-based HD had the highest effectiveness (8.37 QALYs) but with higher cost (USD\$97,917) than the PD group. The incremental cost-effectiveness ratio (ICER) was USD\$16,934 per QALY gained for home-based HD over PD. From the societal perspective, the results were similar and the ICER was USD\$1195 per QALY gained for homebased HD over PD. Both ICERs fell within the acceptable thresholds. Changes in model parameters via sensitivity analyses had a minimal impact on ICER values.

Conclusions: This study assessed the cost-effectiveness of dialysis modalities and service delivery models for ESRD patients under "Peritoneal Dialysis First" policy. For both healthcare provider and societal perspectives, PD as firstline dialysis modality was cost-saving relative to hospital-based HD, supporting the existing PD First or favoured policy. When compared with PD, Nocturnal home Home-based HD was considered a cost-effective first-line dialysis modality for ESRD patients.

Keywords: Cost-effectiveness, Economic burden, End-stage renal disease, Peritoneal dialysis first, Dialysis, Nocturnal home haemodialysis

\footnotetext{
* Correspondence: carlosho@hku.hk

${ }^{1}$ Department of Family Medicine and Primary Care, Li Ka Shing Faculty of Medicine, The University of Hong Kong, Rm 1-01, 1/F, Jockey Club Building for Interdisciplinary Research, 5 Sassoon Road, Pokfulam, Hong Kong, China Full list of author information is available at the end of the article
} 


\section{Background}

With the rising global burden of ESRD, there is a real need for effective and cost-effective renal replacement therapy (RRT) among patients with end-stage renal disease (ESRD) [1]. In Hong Kong where the "Peritoneal Dialysis First" policy [2-4] has been implemented over 25 years, the prevalent cases of patients on peritoneal dialysis (PD) was 3-4 times greater than the those of patients on haemodialysis (HD) [5]. Owing to the increased number of ESRD patients on dialysis and onto transplant waiting list, organs from both living or deceased donors for renal transplantation were scarce over the past decade [6]. Dialysis treatment is a pragmatic first-line treatment option for patients with ESRD. PD is offered as the first-line dialysis treatment to incident dialysis patients in public healthcare sector, whereas HD is offered to patients who have medical contraindications for PD. Another alternative available in Hong Kong is the nocturnal home HD program launched in 2006 [7, 8], commencing HD treatment at home. This option is the least popular, consistent with data from United States Renal Data System, in which only $2.7 \%$ of prevalent dialysis patients received home HD [9].

Health economic evidence demonstrated that renal transplantation was increasingly favoured approach to save costs while conferring additional benefits in clinical effectiveness $[10,11]$. The lack of availability of live or deceased donor organs remains practical challenges to implement renal transplantation at population level. Increasing ratio of $\mathrm{PD} / \mathrm{HD}$ among incident dialysis patients and offering access to dialysis at home are shown to be effective and cost-effective strategies [10-12]. Implementation of former strategy may not be applicable to ESRD management in Hong Kong due to the "Peritoneal Dialysis First" policy. However, health economic value for improving $\mathrm{HD}$ access and switching dialysis treatment venue from in-centre or hospital to home is uncertain. There is therefore a need to conduct an economic evaluation of the cost-effectiveness of available dialysis modalities in managing ESRD patients in the Hong Kong setting which may provide an additional perspective for consideration by other countries with PD first or favoured policy.

The study aims to determine the lifetime cost-effectiveness of three dialysis modalities (PD, hospital-based HD or homebased HD) as first-line treatment for ESRD patients under the "Peritoneal Dialysis First" policy. We hypothesize that the PD strategy is more cost-effective than the hospital-based HD and home-based strategy from health provider's perspective but the home-based HD strategy may be a cost-effective dialysis strategy from societal perspective.

\section{Methods}

\section{Study design}

This study compared cost-effectiveness of three dialysis modalities as first-line treatment strategies, and evaluated lifetime cost-effectiveness analysis of the hospital-based HD strategy or home-based HD strategy in comparison with the currently preferred PD strategy in Hong Kong.

\section{Costing analysis}

Costing analysis was performed from both the health provider and societal perspectives. A micro-costing and bottom approach was applied in the costing analysis [13]. From the health provider's perspective, direct medical costs associated with dialysis and its related health service delivery were taken into consideration. Societal costs were the sum of direct medical costs, direct nonmedical costs (e.g. patients' out-of-pocket costs due to self-prescription and transportation), and indirect costs (e.g. time costs spent by patients and their caregivers). Unit costs of each item were referenced to the Hospital Authority Government Gazette list of health service charges for non-Hong Kong residents in 2017 [14] and the Hospital Authority list of charges for private services in 2017 [15]. Estimation of costs accrued by pre-dialysis surgery, surgery related to the removal of catheter or vascular access, dialysis training and re-training, machine rental and consumables, renal transplantation and post-transplant follow-up, outpatient visits, emergency visits, and hospitalization were entailed in our prior publication [13]. Full list of unit cost of health service items is listed in Table 1.

\section{Health utility measurement}

Long-term cost-effectiveness analysis in terms of QALYs required the use of utility scores, estimated from a previously published systematic review [16], and previous HRQOL surveys conducted by our research team [17, 18]. Utility score of renal transplanted patients was taken from the same systematic review [16]. Comparison of SF-12 scores across three dialysis modalities was performed using pooled data from the current study and a previous study conducted by our research team $[17,18]$. The results of these HRQOL data measured by SF-12 Health Survey, shown to be valid and reliable in Hong Kong [19], were converted to SF-6D utility scores through Hong Kong Chinese population scoring algorithm [20]. The SF-6D utility scores of ESRD undergoing PD, hospital-based HD and home-based HD patients were further converted to disutility (one minus utility) associated with each health state.

\section{Cost-effectiveness analysis}

Cost-effectiveness analysis was performed by using Markov modelling. Empirical data on costing information and health utility scores taken from our previous studies [13, 18] was combined with published data on utility scores $[16,17]$, health state transitions [21], transplantation and 
Table 1 Unit cost of each healthcare service related to patients with end-stage renal disease

\begin{tabular}{|c|c|c|}
\hline Healthcare service & Cost (USD) & Reference \\
\hline General outpatient clinic (per visit) & 57.1 & Hospital Authority [14] \\
\hline Specialist outpatient clinic (per visit) & 152.6 & Hospital Authority [14] \\
\hline Accident and emergency (per visit) & 157.7 & Hospital Authority [14] \\
\hline Hospital stay (per night) & 653.8 & Hospital Authority [14] \\
\hline Haemodialysis (per session) & 384.6 & Hospital Authority [14] \\
\hline $\begin{array}{l}\text { PD home machine rental consumables, maintenance } \\
\text { and insurance (per month) }\end{array}$ & 641.0 & Personal communication with nephrologists \\
\hline Home-based HD machine rental and consumables (per month) & 769.2 & Personal communication with nephrologists \\
\hline \multicolumn{3}{|l|}{ Pre-dialysis access surgery } \\
\hline PD: insertion / removal of peritoneal dialysis catheter & 3192.3 & Hospital Authority [15] \\
\hline HD: Insertion / removal of haemodialysis catheter ${ }^{\text {b }}$ & 2057.7 & Hospital Authority [15] \\
\hline $\begin{array}{l}\text { HD: Arteriovenostomy for renal dialysis (arteriovenous } \\
\text { fistula, arteriovenous graft) }\end{array}$ & 6384.6 & $\begin{array}{l}\text { Hospital Authority [15]. Personal communication } \\
\text { with nephrologists }\end{array}$ \\
\hline Renal transplantation & $10,278.8$ & Hospital Authority [15] \\
\hline \multicolumn{2}{|l|}{ Post-transplant follow-up ${ }^{c}$} & \multirow[t]{3}{*}{ Personal communication with nephrologists } \\
\hline Initial year & 1678.2 & \\
\hline Subsequent years & 610.3 & \\
\hline \multicolumn{2}{|l|}{ Dialysis training ${ }^{a}$} & \multirow[t]{4}{*}{ Personal communication with nephrologists } \\
\hline$P D^{d}$ & 1692.3 & \\
\hline Hospital-based HD & 0 & \\
\hline Home-based HD & $12,184.6$ & \\
\hline \multicolumn{2}{|l|}{ Re-training after peritonitis ${ }^{\mathrm{a}, \mathrm{d}}$} & \multirow[t]{2}{*}{ Personal communication with nephrologists } \\
\hline PD & 1692.3 & \\
\hline \multicolumn{3}{|c|}{ 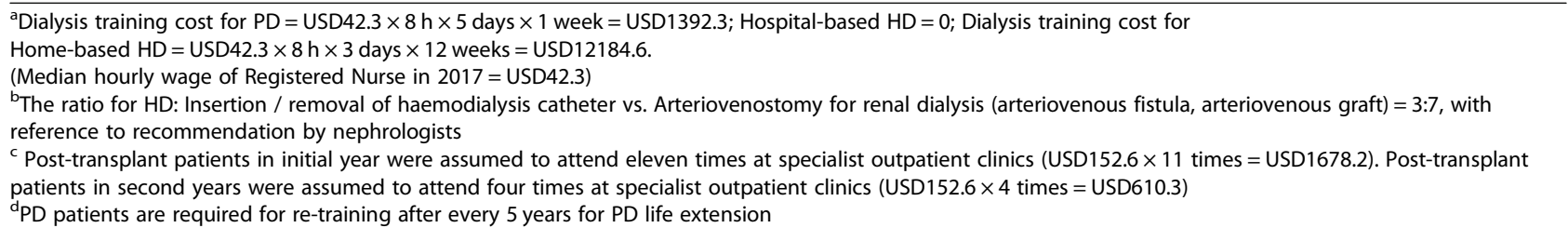 } \\
\hline
\end{tabular}

mortality rates $[5,22,23]$ to estimate long-term effectiveness and cost-effectiveness.

\section{Outcome measures}

Outcomes were total costs and total effectiveness quantified by QALYs of three competing dialysis strategies for ESRD management, and incremental cost-effectiveness ratio of hospital-based HD and home-based HD relative to PD strategy from both the healthcare provider and societal perspectives.

\section{Data analysis}

Three first-line treatment strategies for ESRD patients, i.e. hospital-based HD, home-based HD and PD, were compared in terms of cost and effectiveness using a decision analytic model based on Markov modelling. A hypothetical static cohort of 100,000 individuals requiring RRT was entered into Markov modelling and simulated at age 60 (median age of
ESRD entering into RRT was 59.1 in 2013 [5]) until the terminal states of death by 1 -year cycle. Discounting at an annual rate of 3\% was performed to annual total costs and QALYs before aggregation. The five principal Markov states of ESRD are "Home-based HD", "Hospital-based HD", "PD”, “Transplant and post-transplant", and "Death". To start with, each patient received one of the three initial RRT modalities. For each 1-year cycle, each patient stayed either at the same Markov state or at the transition to a new state based on given transition probabilities. Our model enabled the annual switching among three forms of dialysis, except for the one from home-based HD to PD. Renal transplanted patients would stay at posttransplant health state, and were prohibited from switching to any forms of dialysis. Annual mortality rates depend on current treatment modality, but independent of previous or initial treatment modalities if a change in treatment modality occurs. Patients 
undergoing home-based HD are not at risk of mortality because patients who develop complications are always admitted to hospital and are switched to hospital-based HD before they died. The annual health status transition between different health states and mortalities in Markov modelling is shown in Fig. 1. A summary of the assumptions on the model structure, health status transitions and parameters is presented in Table 2.

Both the deterministic and probabilistic sensitivity analyses were performed to test the uncertainty and robustness of the results to changes in the cost and clinical parameters. Deterministic sensitivity analysis examined the impact of changing each specific parameter on modelling outputs such as mean incremental cost and effectiveness. Probabilistic sensitivity analysis was conducted to achieve a full examination of uncertainly involved in all parameters. All the model parameters except mortality rate were considered in sensitivity analyses because mortality rates for health status were estimated based on empirical data from Hong Kong Renal Registry [3, 5, 22]. Annual healthcare and societal costs in the first and second years of three dialysis groups reported from our previous cost analysis [13] were source of cost parameters for the first and subsequent years of three dialysis groups. Model parameters including cost, disutility and clinical parameters, alongside the range and simulated distribution for sensitivity analyses are listed in Table 3.

The incremental cost-effectiveness ratio (ICER) was calculated by dividing the incremental cost by the incremental effectiveness in terms of QALYs gained for the hospital-based or home-based HD strategy relative to the reference PD strategy. A strategy that is more effective but less costly than the alternative was defined as a cost-saving strategy. A strategy was regarded as costeffective relative to the alternative strategy if its incremental cost-effectiveness ratio is less than the threshold of one to three times gross domestic product per capita according to the cost-effectiveness threshold derived based on opportunity cost for Hong Kong SAR [24]. The Markov model was built using TreeAge Pro Suite (TreeAge Software, Williamstown, MA, USA).

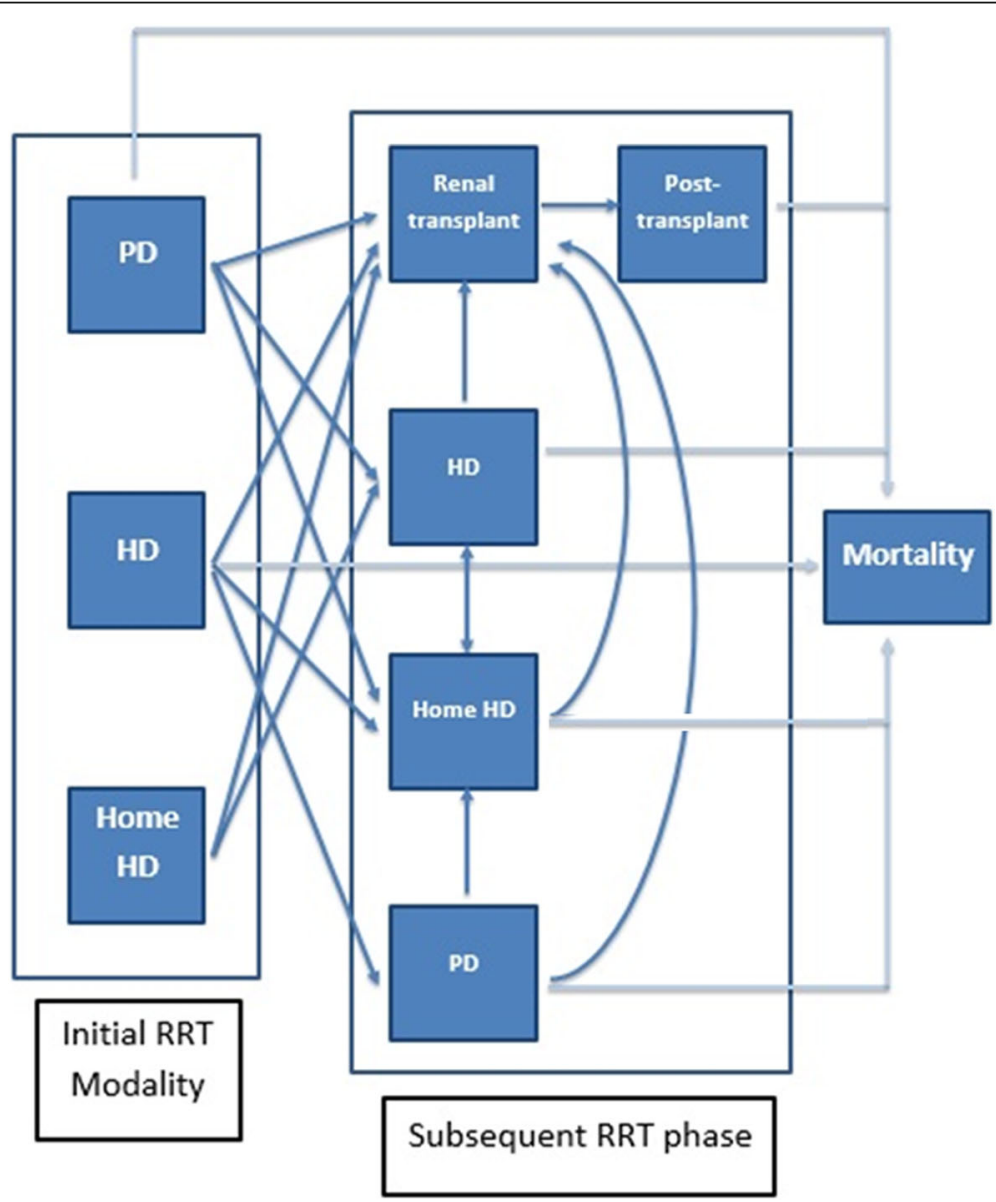

Fig. 1 Annual transition between health states in lifetime simulation 
Table 2 Summary list of assumptions on the model structure, transition and parameters

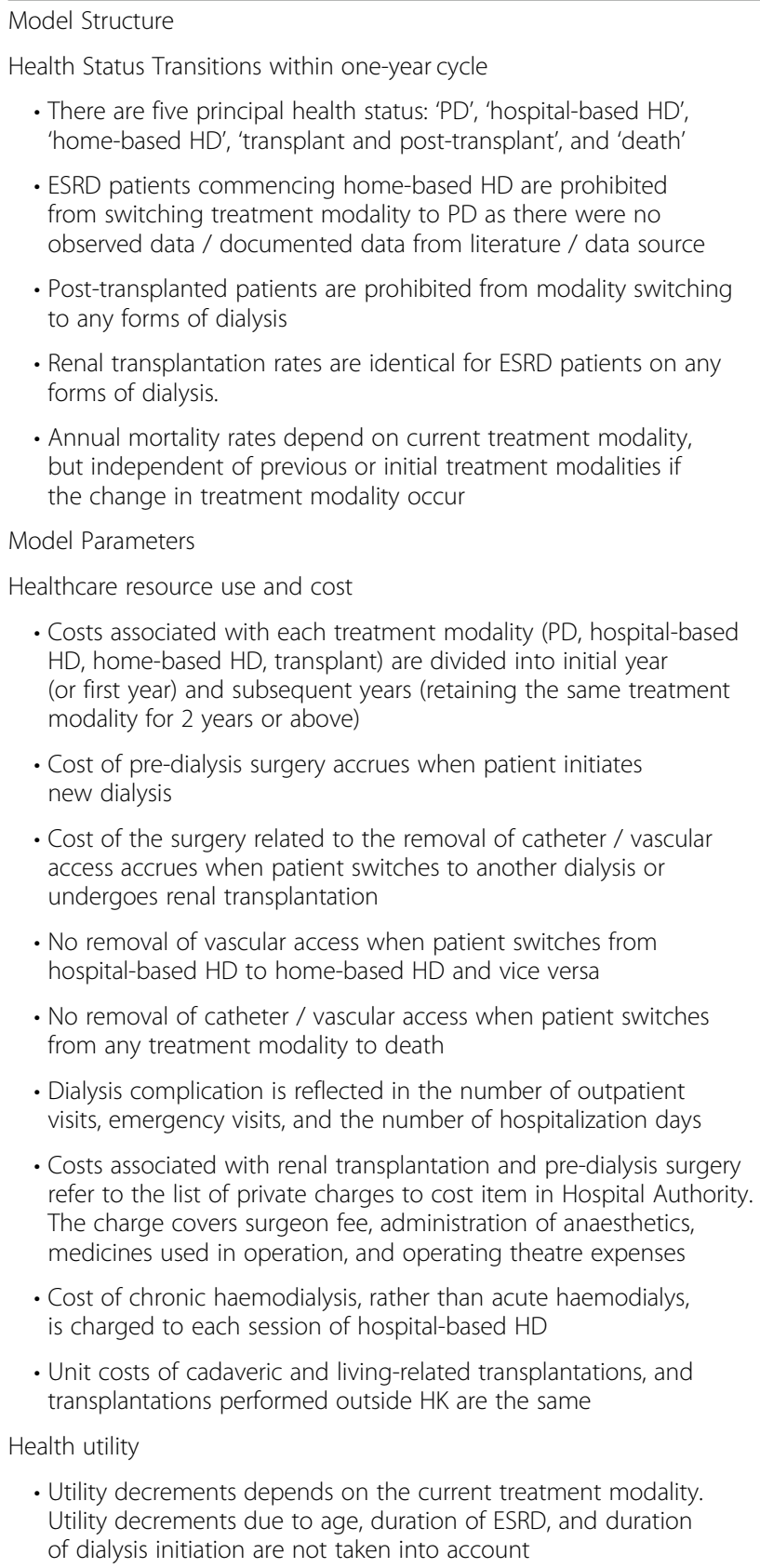

- No removal of catheter / vascular access when patient switches from any treatment modality to death

- Dialysis complication is reflected in the number of outpatient visits, emergency visits, and the number of hospitalization days

- Costs associated with renal transplantation and pre-dialysis surgery refer to the list of private charges to cost item in Hospital Authority. The charge covers surgeon fee, administration of anaesthetics, medicines used in operation, and operating theatre expenses

- Cost of chronic haemodialysis, rather than acute haemodialys, is charged to each session of hospital-based HD

- Unit costs of cadaveric and living-related transplantations, and transplantations performed outside HK are the same

Health utility

- Utility decrements depends on the current treatment modality. Utility decrements due to age, duration of ESRD, and duration of dialysis initiation are not taken into account

\section{Results}

\section{Base-case scenario}

Table 4 shows the cost-effectiveness analysis over the lifetime horizon in base-case scenario from healthcare provider and societal perspectives. From the healthcare provider perspective, hospital-based HD group had higher cost USD $\$ 142,389$ than PD but the lowest effectiveness 6.58 QALYs, and so it was dominated by the PD group (cost $=$ USD $\$ 76,915$; effectiveness =7.13 QALYs). Home-based HD had the highest effectiveness 8.37 QALYs but with higher cost USD\$97,917 than PD group. The ICER of USD\$16,934 per QALY gained for home-based HD over PD. From the societal perspective, the results were similar to that of healthcare provider. The ICER was USD\$1195 per QALY gained for homebased HD over PD. Both ICERs were less than thresholds derived based on opportunity costs.

\section{Deterministic sensitivity analysis}

The impact of changing each specific parameter on the cost-effectiveness results for the modality of Home-based HD versus PD from both the healthcare provider and societal perspectives are shown by tornado diagrams (Fig. 2a and b). Parameters that have the biggest impact are shown at the top of the tornado diagrams, whereas those that have the least impact are shown at the bottom. Both perspectives showed similar results that discount rate and disutility in renal transplant had the greatest impact while disutility in hospital-based $\mathrm{HD}$ and annual cost of hospital-based HD in initial year had the least impact on the cost-effectiveness. All model parameters had a minimal impact on ICER values.

\section{Probabilistic sensitivity analysis}

Cost-effectiveness acceptability curves (Fig. 3a and b) depicts that, from the perspective of healthcare provider, the probabilities of PD, hospital-based HD and homebased HD being the optimal treatment strategy were 40, $0,60 \%$, respectively, using an ICER threshold of USD\$18,609 per QALY. Hospital-based HD was dominated at any ICER thresholds, and the probability of home-based HD being cost-effective was more than that of PD when the ICER threshold was higher than USD $\$ 18,609$ and vice versa. The results from the societal perspective were similar to those from the healthcare provider perspective. The probabilities of $\mathrm{PD}$, hospitalbased HD and home-based HD being the optimal treatment strategy were $54,0,46 \%$, respectively, using an ICER threshold of USD $\$ 18,609$ per QALY. Hospitalbased HD was dominated at any ICER thresholds, and the probability of home-based HD being cost-effective was more than that of PD when the ICER threshold was higher than USD\$18,609 and vice versa.

\section{Discussions}

Accumulative evidence by modelling studies demonstrated that renal transplantation was cost-saving and the most preferable strategy $[10,11,25]$ but the optin consent system and scarcity of organ donations [6] limited the feasibility of renal transplantation as the first-line treatment modality in the management of ESRD patients. Cost-effectiveness analysis assessed the 
Table 3 Parameter values, ranges (95\% confidence interval) and distributions used in the base-case scenario

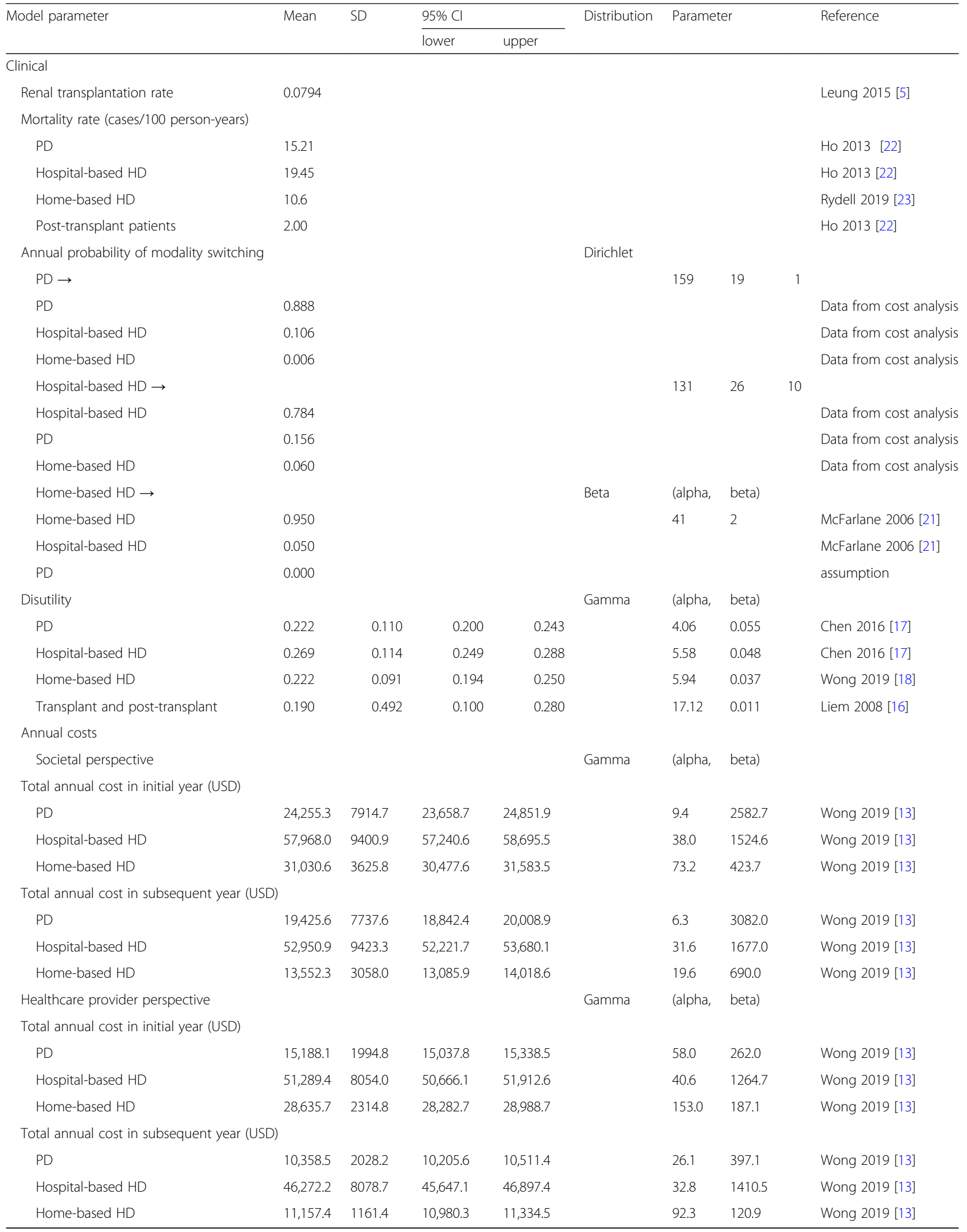

Cl Confidence Interval, SD Standard deviation; 
Table 4 Lifetime cost (USD) of each dialysis modality strategy as first-line treatment and incremental cost-effectiveness ratio in basecase scenario

\begin{tabular}{|c|c|c|c|c|c|c|}
\hline Perspective & Strategy & Cost (USD) & Incremental Cost (USD) & Effectiveness (QALY) & Incremental Effectiveness & ICER (USD / QALY gained \\
\hline \multicolumn{7}{|c|}{ Healthcare provider } \\
\hline & $P D^{a}$ & 76,915 & NA & 7.13 & NA & NA \\
\hline & Home-based HD & 97,917 & 21,002 & 8.37 & 1.24 & 16,934 \\
\hline & Hospital-based HD & 142,389 & 44,472 & 6.58 & Dominated by PD & \\
\hline \multicolumn{7}{|l|}{ Societal } \\
\hline & $P D^{a}$ & 109,668 & NA & 7.13 & NA & NA \\
\hline & Home-based HD & 111,150 & 1482 & 8.37 & 1.24 & 1195 \\
\hline & Hospital-based HD & 166,648 & 56,980 & 6.58 & Dominated by PD & \\
\hline
\end{tabular}

NA Not applicable, QALY quality-adjusted life-year, ICER incremental cost-effectiveness ratio

${ }^{\mathrm{a}} \mathrm{PD}$ is the reference category when calculating incremental cost and incremental effectiveness

total costs and effectiveness for three competing strategies as first-line treatment, and identified the homebased HD strategy as the cost-effective first-line dialysis modality. Over a lifetime horizon based on a simpler cost-minimization approach, the PD strategy was the most preferable first-line dialysis strategy from both healthcare provider and societal perspectives. This finding echoed previous costing analyses based on the context of Hong Kong [2], Asian-Pacific countries [26] and other developed countries [25, 27].

By aggregating annual costs and effectiveness, hospital-based HD strategy accumulated the lowest total health effectiveness and high total costs, mainly attributable to expensive HD sessions in hospital setting. From both perspectives, PD strategy dominated hospital HD strategy. Our priori hypothesis that PD was more cost-effective than hospital-based HD from healthcare provider's viewpoint was supported by our current finding. Such principal finding was in line with priority setting of other developed countries such as UK [12], Sweden [28], Singapore [29] and Taiwan [30], and thus supported the implementation of "Peritoneal Dialysis First" policy. When making pairwise comparisons between PD and home-based $\mathrm{HD}$, the incremental cost-effectiveness ratio was USD\$12,020 and USD\$10,357 per one additional QALY gained from healthcare provider's and societal viewpoints, respectively. Those ICER values were less than the upper bound that derived based on opportunity cost (range $=$ USD $\$ 18,609-20,223$ ) [24]. To interpret the cost-effectiveness of home-based strategy, the decision-making depends on whether the healthcare system and resources are willing to pay incremental costs for suitable ESRD patients for homebased HD. Current finding echoed the cost-utility analysis of hospital HD versus and home HD in the UK setting [31].

With respect to modelling aspects, our simulation model adopted one-yearcycles in line with three previous modelling studies $[10,29,32]$ but a shorter length of cycle such as monthly cycles $[11,12,31]$ and 6-month cycles [28] was utilized in other published studies. This was in part due to the frequent occurrence of dialysis complications and modality switching within a short life expectancy for ESRD patients. By principle, the shorter the Markov cycle, the smaller is the error in the estimates of model outcomes [33], as simulation model is closer to the continuous time transition in reality. However, the choice of cycle length across simulation models reported consistent conclusions for costeffectiveness comparison of PD versus hospital HD, capturing the cost and clinical advantages of $\mathrm{PD}$ strategy in comparison with hospital-based HD.

\section{Limitations}

Our study has several limitations. First, the allocation of dialysis modality was not randomized due to the "Peritoneal Dialysis First" policy so demographic and clinical characteristics of patients varied across three dialysis modalities. Confounded by treatment indications, hospital-based HD patients were more medically complicated, incurring a higher treatment and complication costs [3], and thus might be clinically unfeasible to undergo home-based HD as firstline dialysis modality. Besides, continuous ambulatory peritoneal dialysis and automated peritoneal dialysis were subtypes of peritoneal dialysis, and were not classified as separate RRT strategy in in current analysis. Second, health utility scores applied in modelling were sourced from cross-sectional data primarily. Changes in health utility throughout the remaining lifetime and separation of utility scores between incident and prevalent ESRD patients were not taken into account in the cost-effectiveness model. Thirdly, the annual transition in our analysis relied on the assumption that renal transplantation rates were identical across three modalities, and posttransplant patients were prohibited from modality switch 


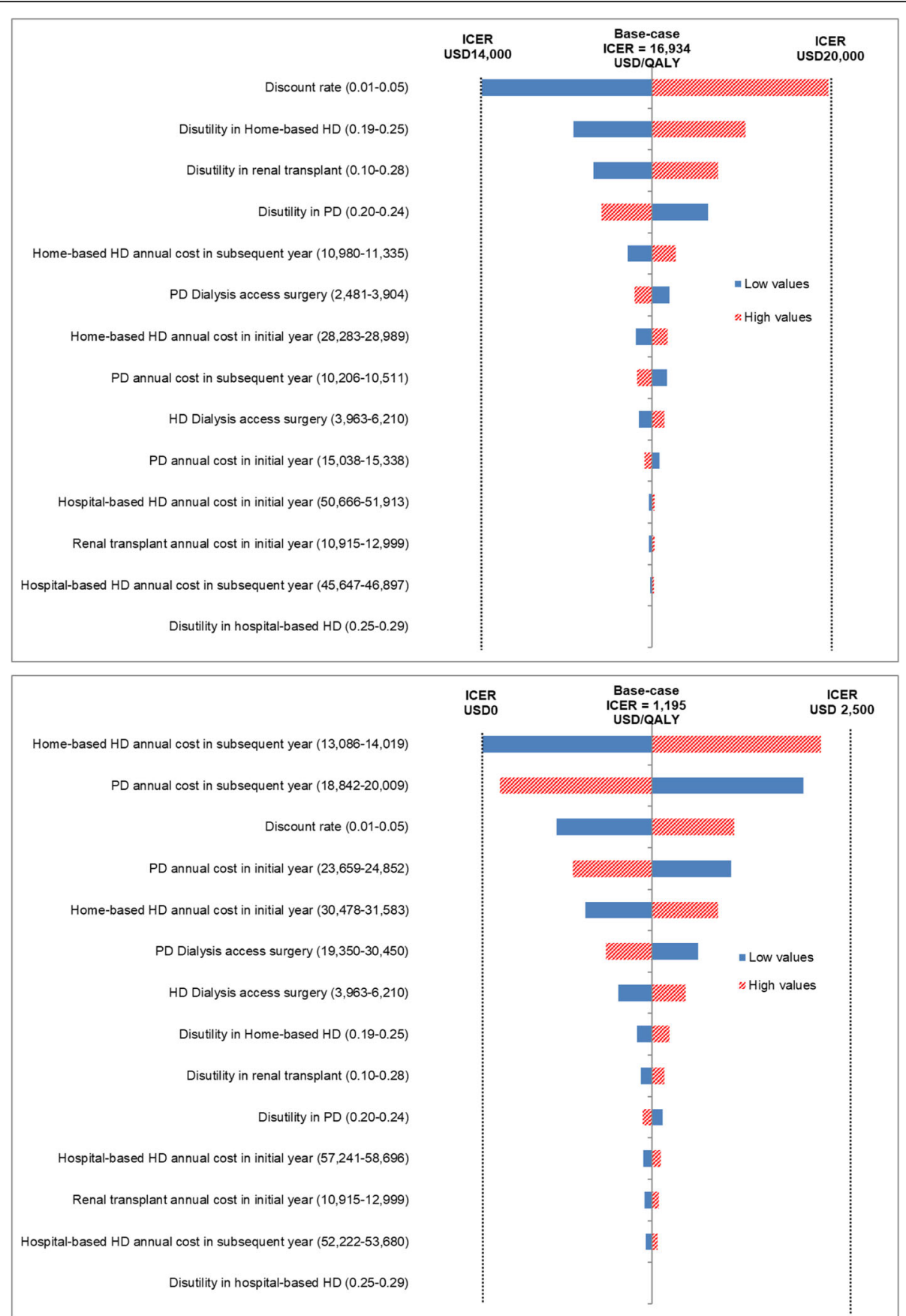

Fig. 2 Tornado diagrams for one-way sensitivity analysis of incremental cost-effectiveness ratio of home-based haemodialysis relative to peritoneal dialysis a) from the healthcare provider perspective and $\mathbf{b}$ ) from the societal perspective

underlining the assumption that dialysis modality had no impact on renal graft. Finally, additional investments such as building capital and overhead costs associated with dialysis were identified in previous costing analyses [25, 32, 34, 35], and were not taken into consideration. Those elements were not possible to be quantified, and thus excluded from costs estimation in current analysis. Given in above, findings from this simulation models for healthcare setting under PD First policy might not be generalizable to healthcare setting in other countries.

\section{Conclusions}

This cost-effectiveness analysis assessed lifetime costs of ESRD patients initiating hospital-based HD, home-based HD or PD dialysis modalities under the 

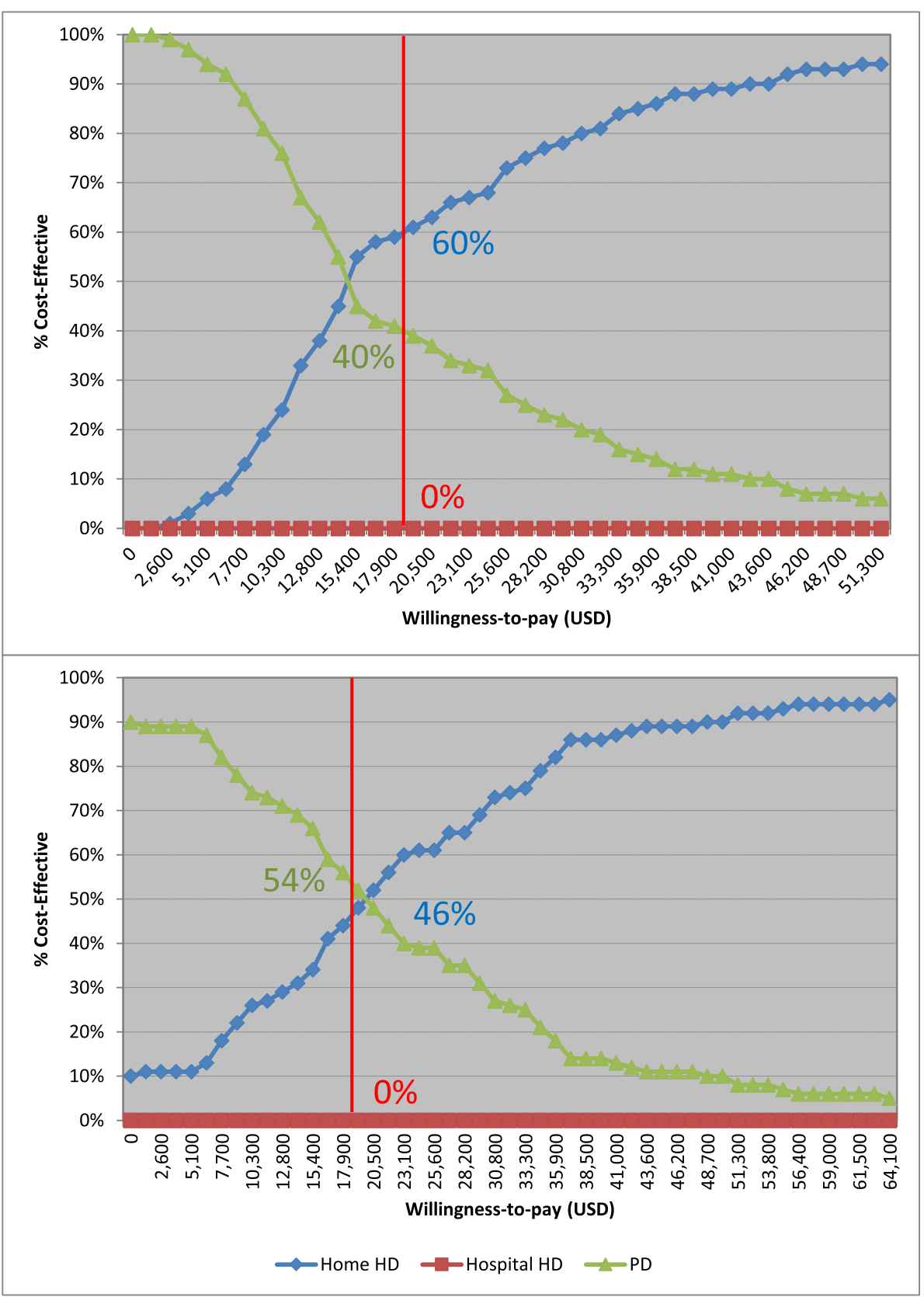

Note: willingness-to-pay threshold of USD \$18,609

Fig. 3 Cost-effectiveness acceptability curve a) from the healthcare provider perspective; and $\mathbf{b}$ ) from the societal perspective

"Peritoneal Dialysis First" policy, and evaluated the cost-effectiveness of three dialysis modalities as firstline treatment from the health provider's and societal viewpoints. Over a simulated lifetime horizon, hospital-based HD was dominated by PD from the healthcare provider perspective and societal perspective when indirect costs were taken into account. Those ICER values fell within threshold derived based on opportunity costs, supporting the PD First or favoured policy. Nocturnal home HD was the most effective strategy but more costly, which can be considered cost-effective for those patients who are suitable for home-based HD. Although there is no informed choice of dialysis under the "Peritoneal Dialysis First" policy, our results may help the general public, nephrologists and health policymakers to make recommendations and decisions about the management of ESRD patients. 


\section{Abbreviations}

ESRD: End-stage renal disease; HD: Haemodialysis; Home HD: Nocturnal home HD; HRQOL: Health-related quality of life; ICER: Incremental costeffectiveness ratio; PD: Peritoneal dialysis; QALY: Quality-adjusted life-year; RRT: Renal replacement therapies; SF-6D: Short-Form Six-Dimension

\section{Acknowledgements}

The authors thank Mr. Ryan Pak and Mr. Chu Wa Ho for administrative support, and Dr. Cindy Choy for valuable input to the design of this study.

\section{Authors' contributions}

C.W. constructed the study design and conducted the data analysis. C.W., M.M., and S.F. were responsible for the paper draft and data interpretation. J.C., S.F., M.M., W.K.L, S.L.L, D.C., Y.L.C., I.K., TC, and C.L. were responsible for expertise advice and final approval. All authors read and approved the final manuscript.

\section{Funding}

This study was supported by the Health and Medical Research Fund (grant number 13142451), Food and Health Bureau, Government of the Hong Kong SAR. The funder had no role in study design, data collection and analysis, decision to publish, or preparation of the manuscript.

\section{Availability of data and materials}

The data that support the findings of this study are available from database of the Clinical Management System of the Hospital Authority, Hong Kong but restrictions apply to the availability of these data, which were used under license for the current study, and so are not publicly available. Data are however available from the authors upon reasonable request and with permission of Hospital Authority, Hong Kong.

\section{Ethics approval and consent to participate}

Research ethics approval was obtained from the institutional review board before patient recruitment (HKU/Hospital Authority Hong Kong West IRB reference number: UW 15-592; KWC-REC reference KW/EX-16-011(95-11); Joint CUHK-New Territories East Cluster reference: CREC 2015.726).

\section{Consent for publication}

Not applicable.

\section{Competing interests}

The authors declare that they have no competing interests.

\section{Author details}

'Department of Family Medicine and Primary Care, Li Ka Shing Faculty of Medicine, The University of Hong Kong, Rm 1-01, 1/F, Jockey Club Building for Interdisciplinary Research, 5 Sassoon Road, Pokfulam, Hong Kong, China. ${ }^{2}$ Bau Institute of Medical and Health Sciences Education, Li Ka Shing Faculty of Medicine, The University of Hong Kong, Hong Kong, China. ${ }^{3}$ Department of Medicine and Geriatrics, Princess Margaret Hospital, Hong Kong, China. ${ }^{4}$ Division of Nephrology, Department of Medicine, Tung Wah Hospital, Hong Kong, China. ${ }^{5}$ Department of Medicine, Alice Ho Miu Ling Nethersole Hospital, Hong Kong, China. ${ }^{6}$ Division of Nephrology, Department of Medicine, Li Ka Shing Faculty of Medicine, The University of Hong Kong, Hong Kong, China.

Received: 24 April 2019 Accepted: 29 January 2020

Published online: 04 February 2020

\section{References}

1. Li PK-T, Chow KM, Van de Luijtgaarden MWM, Johnson DW, Jager KJ, Mehrotra R, Naicker S, Pecoits-Filho R, Yu XQ, Lameire N. Changes in the worldwide epidemiology of peritoneal dialysis. Nat Rev Nephrol. 2017;13(2): 90-103.

2. Yu AWY, Chau KF, Ho YW, Li PKT. Development of the "peritoneal dialysis first" model in Hong Kong. Perit Dial Int. 2007;27(Suppl 2):S53-5.

3. PK-t L, Chow KM. Peritoneal Dialysis-first policy made successful: perspectives and actions. Am J Kidney Dis. 2013;62(5):993-1005.

4. Choy AS, Li PK. Sustainability of the peritoneal Dialysis-first policy in Hong Kong. Blood Purif. 2015;40(4):320-5.
5. Leung CB, Cheung WL, Li PK. Renal registry in Hong Kong-the first 20 years. Kidney Int Suppl. 2015;5(1):33-8.

6. Department of Health, Government of the HKSAR: Statistics ( Milestones of Hong Kong Organ Transplantation ). https://www.organdonation.gov.hk/ eng/statistics.html.

7. Tang HL, Tang CM, Chu KH, Lee W, Cheuk A, Yim KF, Fung KS, Chan HW, Tong KL. The first nocturnal home haemodialysis patient in Hong Kong. Hong Kong Med J. 2008;14(5):395-8.

8. Tang HL, Wong JH, Poon CK, Tang CM, Chu KH, Lee W, Fung SK, Chau KF, Li CS, Tong KL. One year experience of nocturnal home haemodialysis with an alternate night schedule in Hong Kong. Nephrology (Carlton). 2011;16(1):57-62.

9. United States Renal Data System: Chapter 13: International Comparisons. 2016. https://www.usrds.org/2016/view/v2_13.aspx.

10. Howard K, Salkeld G, White S, McDonald S, Chadban S, Craig JC, Cass A. The cost-effectiveness of increasing kidney transplantation and home-based dialysis. Nephrology. 2009;14(1):123-32.

11. Haller M, Gutjahr G, Kramar R, Harnoncourt F, Oberbauer R. Costeffectiveness analysis of renal replacement therapy in Austria. Nephrol Dial Transplant. 2011;26(9):2988-95.

12. Treharne C, Liu FX, Arici M, Crowe L, Farooqui U. Peritoneal dialysis and inCentre haemodialysis: a cost-utility analysis from a UK payer perspective. Appl Health Econ Health Policy. 2014;12(4):409-20.

13. Wong $\mathrm{CKH}$, Chen JY, Fung SKS, Mok MMY, YI C, Kong I, Lo WK, Lui SL, DTM C, CLK L. Direct and indirect costs of end-stage renal disease patients in the first and second years after initiation of nocturnal home haemodialysis, hospital haemodialysis and peritoneal dialysis. Nephrol Dial Transplant. 2019; 34(9):1565-76.

14. Hospital Authority. Hospital Authority Ordinance (Chapter 113): Revisions to List of Charges, G.N. 3884; 2017.

15. Hospital Authority. List of private services - operations; 2017. http://www3. ha.org.hk/fnc/Operations.aspx?lang=ENG.

16. Liem YS, Bosch $J$, Hunink MG. Preference-based quality of life of patients on renal replacement therapy: a systematic review and meta-analysis. Value Health. 2008;11(4):733-41.

17. Chen JY, Choi EPH, Wan EYF, Chan AKC, Tsang JPY, Chan KHY, Lo WK, Lui SL, Chu WL, Lam CLK. Validation of the disease-specific components of the kidney disease quality of Life-36 (KDQOL-36) in Chinese patients undergoing maintenance Dialysis. PLoS One. 2016;11(5):e0155188,

18. Wong CKH, Chen JY, Fung SKS, Lo WK, Lui SL, Chan DTM, YI C, Kong I, EYF W, CLK L. Health-related quality of life and health utility of Chinese patients undergoing nocturnal home Haemodialysis in comparison to other modes of Dialysis. Nephrology. 2019;24(6):630-7.

19. Lam ETP, Lam CLK, Fong DYT, Huang WW. Is the SF-12 version 2 health survey a valid and equivalent substitute for the SF-36 version 2 health survey for the Chinese? J Eval Clin Pract. 2013;19(1):200-8.

20. McGhee SM, Brazier J, Lam CLK, Wong LC, Chau J, Cheung A, Ho A. Qualityadjusted life years: population-specific measurement of the quality component. Hong Kong Med J. 2011;17(Suppl 6):17-21.

21. McFarlane PA, Bayoumi AM, Pierratos A, Redelmeier DA. The impact of home nocturnal hemodialysis on end-stage renal disease therapies: a decision analysis. Kidney Int. 2006:69(5):798-805.

22. Ho YW, Chau KF, Choy BY, Fung KS, Cheng YL, Kwan TH, Wong PN, Lai WM, Yuen SK, Lo HKS, et al. Hong Kong renal registry report 2012. Hong Kong J Nephrol. 2013;15(1):28-43.

23. Rydell $H$, Ivarsson $K$, Almquist $M$, Segelmark M, Clyne N. Improved long-term survival with home hemodialysis compared with institutional hemodialysis and peritoneal dialysis: a matched cohort study. BMC Nephrol. 2019;20(1):52

24. Woods B, Revill P, Sculpher M, Claxton K. Country-level cost-effectiveness thresholds: initial estimates and the need for further research. Value Health. 2016;19(8):929-35

25. Villa G, Rodríguez-Carmona A, Fernández-Ortiz L, Cuervo J, Rebollo P, Otero A, Arrieta J. Cost analysis of the Spanish renal replacement therapy programme. Nephrol Dial Transplant. 2011;26(11):3709-14.

26. Li PK, Cheung WL, Lui SL, Blagg C, Cass A, Hooi LS, Lee HY, Locatelli F, Wang T, Yang CW, et al. Increasing home based dialysis therapies to tackle dialysis burden around the world: a position statement on dialysis economics from the 2 nd Congress of the International Society for Hemodialysis. Nephrology (Carlton). 2011:16(1):53-6.

27. Baboolal K, McEwan P, Sondhi S, Spiewanowski P, Wechowski J, Wilson K. The cost of renal dialysis in a UK setting - a multicentre study. Nephrol Dial Transplant. 2008;23(6):1982-9. 
28. Sennfalt K, Magnusson M, Carlsson P. Comparison of hemodialysis and peritoneal dialysis--a cost-utility analysis. Perit Dial Int. 2002;22(1):39-47.

29. Yang F, Lau T, Luo N. Cost-effectiveness of haemodialysis and peritoneal dialysis for patients with end-stage renal disease in Singapore. Nephrology. 2016;21(8):669-77.

30. Chang YT, Hwang JS, Hung SY, Tsai MS, Wu JL, Sung JM, Wang JD. Costeffectiveness of hemodialysis and peritoneal dialysis: a national cohort study with 14 years follow-up and matched for comorbidities and propensity score. Sci Rep. 2016;6:30266.

31. Liu FX, Treharne C, Arici M, Crowe L, Culleton B. High-dose hemodialysis versus conventional in-center hemodialysis: a cost-utility analysis from a UK payer perspective. Value Health. 2015;18(1):17-24.

32. Villa G, Fernandez-Ortiz L, Cuervo J, Rebollo P, Selgas R, Gonzalez T, Arrieta J. Cost-effectiveness analysis of the Spanish renal replacement therapy program. Perit Dial Int. 2012;32(2):192-9.

33. Sonnenberg FA, Beck JR. Markov models in medical decision making:a practical guide. Med Decis Mak. 1993;13(4):322-38.

34. Komenda P, Gavaghan MB, Garfield SS, Poret AW, Sood MM. An economic assessment model for in-center, conventional home, and more frequent home hemodialysis. Kidney Int. 2012;81(3):307-13.

35. Agar JW, Knight RJ, Simmonds RE, Boddington JM, Waldron CM, Somerville CA. Nocturnal haemodialysis: an Australian cost comparison with conventional satellite haemodialysis (review article). Nephrology. 2005;10(6): $557-70$

\section{Publisher's Note}

Springer Nature remains neutral with regard to jurisdictional claims in published maps and institutional affiliations.

Ready to submit your research? Choose BMC and benefit from:

- fast, convenient online submission

- thorough peer review by experienced researchers in your field

- rapid publication on acceptance

- support for research data, including large and complex data types

- gold Open Access which fosters wider collaboration and increased citations

- maximum visibility for your research: over $100 \mathrm{M}$ website views per year

At $\mathrm{BMC}$, research is always in progress.

Learn more biomedcentral.com/submissions 